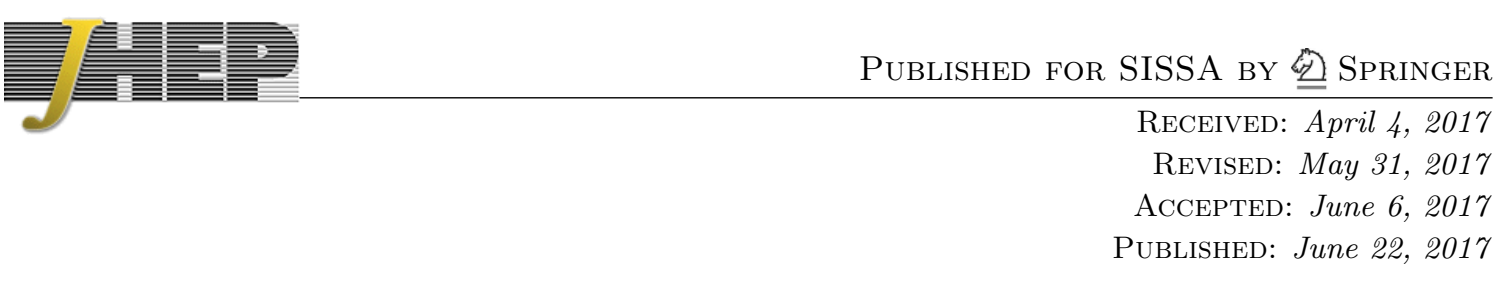

\title{
Closing the window for compressed dark sectors with disappearing charged tracks
}

\author{
Rakhi Mahbubani, ${ }^{a, b}$ Pedro Schwaller ${ }^{c}$ and José Zurita ${ }^{d, e}$ \\ ${ }^{a}$ Theoretical Physics Department, \\ CERN, Geneva, Switzerland \\ ${ }^{b}$ Theoretical Particle Physics Laboratory, Institute of Physics, EPFL, \\ Lausanne, Switzerland \\ ${ }^{c}$ PRISMA Cluster of Excellence 83 Mainz Institute for Theoretical Physics, \\ Johannes Gutenberg University, \\ 55099 Mainz, Germany \\ ${ }^{d}$ Institute for Nuclear Physics (IKP), Karlsruhe Institute of Technology, \\ Hermann-von-Helmholtz-Platz 1, D-76344 Eggenstein-Leopoldshafen, Germany \\ ${ }^{e}$ Institute for Theoretical Particle Physics (TTP), Karlsruhe Institute of Technology, \\ Engesserstraße 7, D-76128 Karlsruhe, Germany \\ E-mail: rakhi@cern.ch, pedro.schwaller@uni-mainz.de, \\ jose.zurita@kit.edu
}

ABSTRACT: We investigate the sensitivity at current and future hadron colliders to a heavy electrically-charged particle with a proper decay length below a centimetre, whose decay products are invisible due to below-threshold energies and/or small couplings to the Standard Model. A cosmologically-motivated example of a framework that contains such a particle is the Minimal Supersymmetric Standard Model in the limit of pure Higgsinos. The current hadron-collider search strategy has no sensitivity to the upper range of pureHiggsino masses that are consistent with the thermal relic density, even at a future collider with $100 \mathrm{TeV}$ centre-of-mass energy. We show that performing a disappearing track search within the inner $10 \mathrm{~cm}$ of detector volume would improve the reach in lifetime by a factor of 3 at the $14 \mathrm{TeV}$ LHC and a further factor of 5 at a $100 \mathrm{TeV}$ collider, resulting in around 10 events for $1.1 \mathrm{TeV}$ thermal Higgsinos. In order to include the particles with the largest boost in the analysis, we furthermore propose a purely track-based search in both the central and forward regions, each of which would increase the number of events by another factor of 5, improving our reach at small lifetimes. This would allow us to definitively discover or exclude the experimentally-elusive pure-Higgsino thermal relic at a $100 \mathrm{TeV}$ collider. Our results illustrate the importance of varying detector design when assessing the reach of future high energy colliders.

Keywords: Beyond Standard Model, Supersymmetric Standard Model

ARXIV EPRINT: 1703.05327 


\section{Contents}

1 Introduction 1

2 Simplified model 3

3 Disappearing charged tracks $\quad 4$

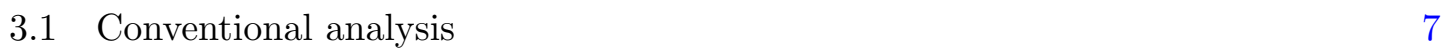

$\begin{array}{lll}3.2 & \text { Track-based (TB) analysis } & 11\end{array}$

$\begin{array}{lll}3.3 & \text { LHC14-HL sensitivity } & 13\end{array}$

4 Conclusions $\quad 14$

$\begin{array}{ll}\text { A Decays and lifetimes } & 17\end{array}$

$\begin{array}{ll}\text { B Impact of transverse cuts on forward track-based analysis } & 18\end{array}$

\section{Introduction}

In spite of the continued shattering of performance goals at the LHC, these are unexpectedly lean times for particle phenomenologists. The wealth of interesting excesses over the Standard Model (SM) that were anticipated, and indeed prepared for, in the run-up to data-taking, have failed to materialize. In their absence the attention of some part of the community has turned to possible signatures of new physics that may be overlooked by our current experimental program. On closer examination this avenue has been extremely fertile, and is now seen to be linked to some of the more exotic examples of physics Beyond the Standard Model (BSM), such as Hidden Valleys [1], or Neutral Naturalness [2, 3], ideas that are currently enjoying a renaissance as a result of our reduced circumstances.

One does not need to resort to exotic models, however, to find signatures that fall through holes in our experimental net. Even our favourite scenarios for BSM physics can yield these, in regions of parameter space that remain relatively unexplored due in no small part to a strong theory prior. As our biases are eroded by data, this less-charted territory merits a more thorough exploration to ensure these gaps in coverage are filled in. A $100-\mathrm{TeV}$ collider is, in that sense, tabula rasa; it provides us the opportunity to build in sensitivity for unconventional signals that are an afterthought at established experimental programs.

An example receiving some attention of late is compressed scenarios, with lowest-lying new states that are near-degenerate in mass. This could lead to long-lived intermediates through kinematic suppression of decay widths, as well as decay by-products that are below the energy threshold for detection. One means of probing such states is by direct measurement of the track due to an electrically-charged parent, with accessible particle 
lifetimes set by the characteristic size of the detecting apparatus. Hence, unlike the Energy and Intensity frontiers, pushing this 'Lifetime Frontier' [4] does not necessarily require the design and construction of an entire experimental facility, but rather moves the focus to detectors and their design; either through the building of a single-purpose long-lived particle detector, as proposed in [4], or adapting a multipurpose detector so it is better suited to measuring a larger range of lifetimes. It is the latter that concerns us here, specifically in relation to electrically-charged particles that are too short-lived $(c \tau \lesssim \mathcal{O}(\mathrm{cm}))$ to leave a conventionally-measurable track at current particle detectors.

The investigation of intermediate-lifetime particles is by no means a modern endeavour. Indeed much of the BSM physics program at the LHC relies on being able to tag a bottom quark due to the mm-scale decay length of the $B$-hadron it results in. In this case, however, the existence of the $B$-hadron is inferred by extrapolation of the tracks of its decay products to a displaced vertex, rather than a measurement of the properties of the parent particle itself. The latter will be indispensable to probe examples where the daughter particles are undetectable, either because they have very little energy, or because they are noninteracting components of a 'dark sector'.

A canonical example of a model containing such a particle is the decidedly un-exotic Minimal Supersymmetric Standard Model (MSSM), whose neutralino sector provides various well-motivated candidates for thermal relic dark matter. Pure Wino and Higgsino states have charged components that are heavier than neutral ones by electroweak loop effects; these $\mathcal{O}(100 \mathrm{MeV})$ splittings result in $c \tau \sim$ mm-cm. Recent work [5, 6] has shown the entire range of viable thermal Wino masses to be discoverable at a $100 \mathrm{TeV}$ collider in this channel. However this is not the case for pure Higgsinos, which have charged-neutral splittings that are twice as large, the decay width being strongly dependent on the splitting. Pure-Higgsino dark matter is also particularly difficult to access directly by other means, since its tiny indirect and direct detection cross sections are beyond even the projected sensitivity of any dark matter experiment currently under consideration.

In this work, we explore the dependence of the reach for such intermediate-lifetime charged particles, on the tracker properties at a hadron colliders, using the disappearing track signature. ${ }^{1}$ Unlike many existing searches for compressed electroweak-charged states [11-26], we operate under the assumption that no information can be obtained from their decay products, making us less sensitive to the origin and properties of the parent. We then express our results in the parameter space of thermal Higgsino dark matter, and show that full coverage of the elusive pure-Higgsino region $\left(m_{\chi} \lesssim 1.1 \mathrm{TeV}\right)$ can be achieved with a total integrated luminosity of $3000 \mathrm{fb}^{-1}$. While our main focus is a $100 \mathrm{TeV}$ protonproton collider (FCC-hh), we also examine similar upgrades to ATLAS and CMS that could improve the LHC reach for compressed Higgsinos at its high-luminosity run (LHC14-HL). In a companion paper [27] we study the reach in the di-lepton plus missing transverse energy channel, which doesn't assume the presence of an electrically-charged state, but relies instead on additional weak radiation from the initial state, in the form of a leptonic $Z$-boson.

\footnotetext{
${ }^{1}$ For other work on long-lived electrically charged particles at the LHC, see [7-10].
} 


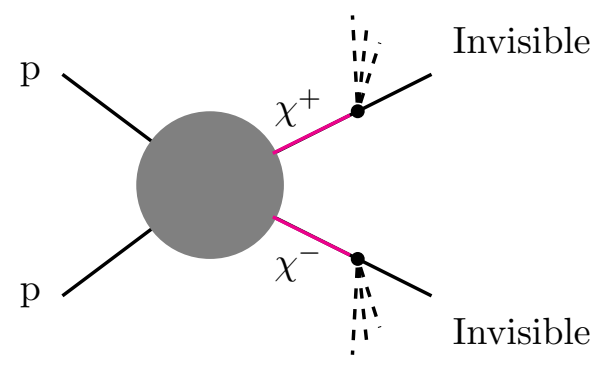

Figure 1. Production of a charged state with proper lifetime $\tau \lesssim 1$ ns and decay products that are invisible at colliders will lead to a charged track that ends ('disappears') within the extent of a tracker subsystem.

\section{Simplified model}

Our disappearing track search will be relevant to any scenario containing a charged particle with proper lifetime $\tau$ below 10 picoseconds, and whose decay products are invisible, either due to small energies or small couplings to the SM, see figure 1. Such states are too short-lived to be covered by conventional disappearing track searches at current [28, 29] or future [5] colliders. We attribute to the charged state a 'nominal decay length' $c \tau$, which translates into an average lab-frame decay length of $\beta \gamma c \tau$ for a particle with velocity $\beta=v / c$ and Lorentz boost $\gamma$. Converting this to an actual charged track length requires us to take into account the Poissonian nature of the decay process, and weight the decay length by the probability that the chargino will travel a distance $d$ without decaying, given by

$$
\mathcal{P}(d)=\exp \left(-\frac{d}{\beta \gamma c \tau}\right) .
$$

We carry out our simulation and analysis within a specific framework containing such a particle, where the usual Standard Model field content is supplemented with a new vectorlike weak-doublet fermion with hypercharge $1 / 2, \chi=\left(\chi^{+}, \chi^{0}\right)$ and Dirac mass $m_{\chi}$ :

$$
\mathcal{L} \supset i \bar{\chi}\left(\not \partial-i g W-i g^{\prime} \frac{1}{2} Y \not B\right) \chi+m_{\chi} \bar{\chi} \chi
$$

where we assume that the neutral component $\chi^{0}$ is stable on collider timescales. Electroweak loops will raise the mass of the charged component, giving rise to a charged-neutral splitting $\Delta_{+}=m_{\chi^{+}}-m_{\chi} \sim \mathcal{O}\left(\alpha m_{Z}\right)$. The precise value of this one-loop effect is specific to the quantum numbers of the multiplet in question, and for the weak doublet is $\sim 360 \mathrm{MeV}$ for $m_{Z} \ll m_{\chi}[30,31]$, yielding a nominal decay length of $6.6 \mathrm{~mm}$. This corresponds to the pure-Higgsino limit of the MSSM, with all other superparticles decoupled.

The decay width of the charged component of the doublet is strongly dependent on the splitting $\Delta_{+}$, as is evident from the expressions for the partial decay widths in appendix A. Since we focus on the case where any SM by-products of the decay are too soft to be detected, we are insensitive to the identity of the daughter particles. As a result we simply allow the nominal decay length of the charged component to be a free parameter, in order to 


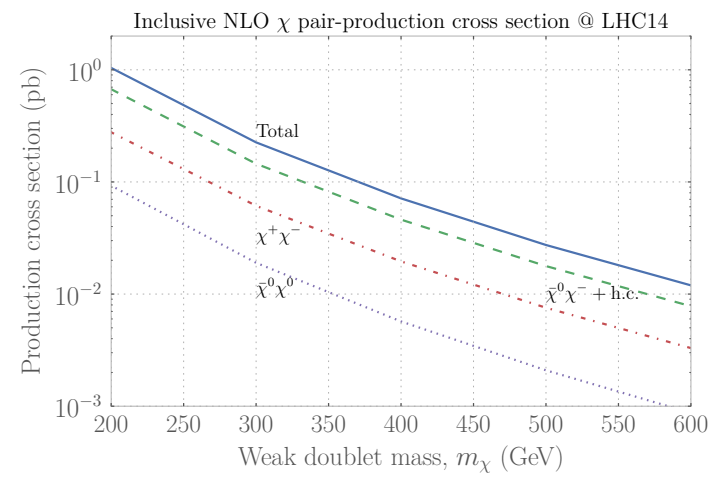

(a)

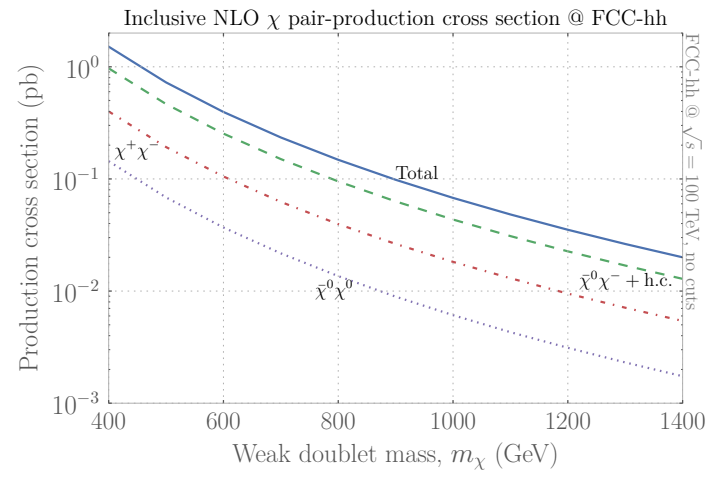

(b)

Figure 2. Pair-production cross sections for a weak doublet with hypercharge $1 / 2$ at NLO, obtained using PROSPINO in the pure-Higgsino limit, for LHC14 (a) and FCC-hh (b).

take into account possible additional contributions to the splitting from couplings to heavy states. $^{2}$ A proper lifetime of 1-10 picoseconds corresponds to a nominal decay length in the mm-cm range.

If we further assume that the neutral component $\chi_{0}$ is stable on cosmological timescales, $\chi$ would be natural thermal relic, saturating the measured relic density for a mass $m_{\chi} \approx 1.1 \mathrm{TeV} .{ }^{3}$ In this work we will remain agnostic about the dark matter connection, and scan over masses beyond this range, although we will use the parameters $m_{\chi}=1.1 \mathrm{TeV}$ and $c \tau=6.6 \mathrm{~mm}$ as references throughout.

We simulate inclusive pair-production of weak doublet $\chi$ using MadGraph5_aMC@NLO [32], matching up to two extra jets using the MLM scheme [33], and shower using Pythia 6.4 [34] with $k_{t}$-showers turned on. Events were clustered with FastJet [35] and analysed with the help of MadAnalysis 5 [36-38]. We normalize the production rate to the NLO cross sections computed with PROSPINO [39] for pure-Higgsino pair production; these yield enhancements of 10-30\% over the Born-level values, and are shown in figure 2 for (a) LHC14 and (b) FCC-hh.

\section{Disappearing charged tracks}

It is clear from the outset that the boost of the charged particle will be a crucial factor in detecting states with nominal decay length below a centimetre. Indeed, if this particle is directly produced, reconstructing its track using tracking elements situated at tens of centimetres from the interaction point would require on average a boost factor $\gamma \beta \sim \mathcal{O}(100)$ were the Poissonian nature of the decay process not taken into account. As it stands, with

\footnotetext{
${ }^{2}$ These could be parametrized, for example, by the Wilson coefficient of a dimension-5 operator $\bar{\chi} \sigma^{a} \chi H^{\dagger} \sigma^{a} H$ for Pauli matrices $\sigma$, coming from integrating out a heavy weak-triplet scalar.

${ }^{3}$ We would also need to introduce a small Majorana splitting between the two neutral components in order to remain consistent with null results from direct detection experiments, a vector-like coupling with the $Z$ being long excluded. However the necessary splitting is small compared with the charged-neutral splitting $\Delta_{+}$, and as such can be neglected when considering the relic density and collider phenomenology.
} 


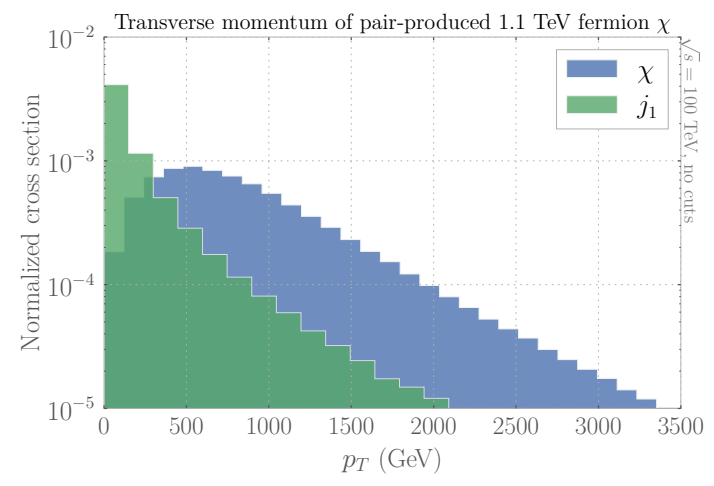

(a)

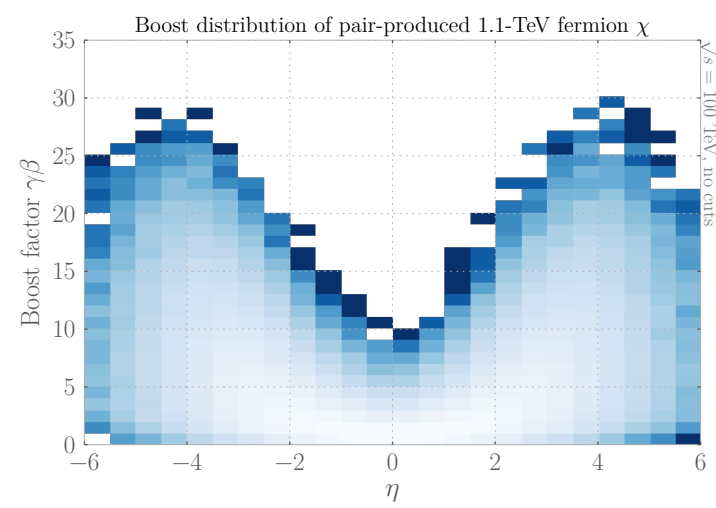

(c)

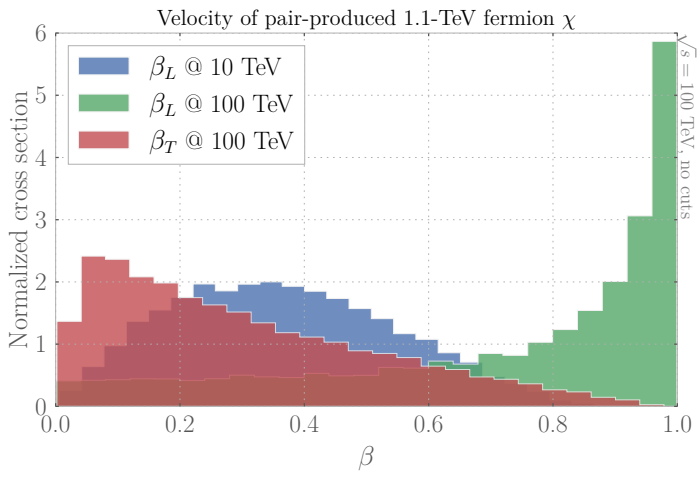

(b)

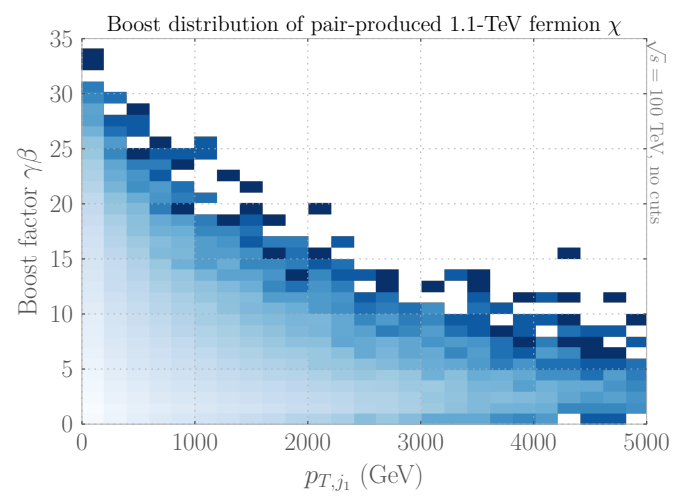

(d)

Figure 3. Kinematic distributions (normalized to unity) for pair-produced weak-doublet fermions $\chi$ with Dirac mass $m \chi=1.1 \mathrm{TeV}$ at FCC-hh. We show 1-dimensional histograms for (a) the $p_{T}$ spectra of the pair-produced particle and leading jet and (b) the transverse and longitudinal beta distributions $\left(\beta_{T}\right.$ and $\left.\beta_{L}\right)$ of the pair-produced particle, with $\beta_{L}$ at $10 \mathrm{TeV}$ centre-of-mass for comparison. We also show correlations between the boost factor $\gamma \beta$ of $\chi$ and (c) $\chi$ pseudorapidity $\eta$ and (d) the $p_{T}$ of the leading jet, with lighter regions signalling a higher density. Details of the model and simulation can be found in the text.

such a large centre-of-mass energy at our disposal, neither large boost nor large statistics should be a limiting factor for $\mathrm{TeV}$-scale particle searches at $100 \mathrm{TeV}$ centre-of-mass.

There is an inherent limitation, however, on the size of the transverse boost: the transverse momentum of a pair-produced particle at a hadron collider is roughly set by the particle mass, as illustrated in figure $3(\mathrm{a})$ for $m_{\chi}=1.1 \mathrm{TeV}$. By contrast, we can infer from the velocity $(\beta)$ distributions in figure $3(\mathrm{~b})$ that the particle mass is negligible on the scale of the longitudinal momentum, resulting in charged particles with near-relativistic longitudinal velocities $\left(\beta_{L}\right)$. The far more modest transverse velocity $\left(\beta_{T}\right)$ distribution, as well as the $\beta_{L}$ distribution for non-negligible mass, are shown for comparison. Hence for a fixed nominal decay length, the particles with the longest lab-frame decay length, corresponding to the those with the largest boost, are predominantly found in the forward direction, see figure $3(\mathrm{c})$, in a pseudorapidity region which we will see is not covered in 
existing disappearing track searches. The boosts in the central region are more moderate, although the particle density is larger (lighter region in the figure). Both these properties will play an important role in our analysis.

The more challenging problem for existing disappearing track searches, which have generic dark matter searches at their core, becomes immediately apparent on examining figure 3(d). With little else to work with, in events with pair-produced dark-sector particles we usually rely on a visible radiated particle $X$ (could be a jet, photon, $Z$-boson, Higgs,...) recoiling off the dark pair with large $p_{T}$. This approach is counterproductive in a disappearing track search. In requiring a minimum recoil $p_{T}$, we are effectively excluding from the analysis those charged particles with the longest lab-frame decay length: for a fixed available energy, the more energetic the recoil state the less energy is available for boosting the charged particles. ${ }^{4}$ To fully benefit from the availability of highly-boosted particles will require a radical change in the philosophy of disappearing track searches, which currently rely almost exclusively on $X p_{T}$ (and related quantities such as missing transverse energy (MET)).

In order to discard recoil $p_{T}$-based quantities a viable alternative must be found. Fortunately the most interesting events in this particular search have a characteristic feature which is rather unusual: a charged track with extremely high momentum, a quantity which could potentially be used as replacements for jet $p_{T}$. Track $p_{T}$ is already used to define signal regions in the current ATLAS search [28], but this measurement could also be combined with track pseudo-rapidity to obtain total track momentum. By setting the boost of the charged particle, track momentum is also directly correlated with the average track length in the lab frame.

However it is impossible to know how precisely this quantity can be measured, particularly for short tracks, or whether this measurement can be made 'on-the-fly'. Moreover, since there exist electrically-charged QCD bound states with similar lifetimes, the hadronic background in the absence of a MET cut will likely be not insignificant. Since we currently have no data on the momentum spectra of such backgrounds, we will be unable to give a quantitative assessment of the sensitivity of an analysis based on this quantity. However we can still make a rough comparison with a more conventional analysis on the basis of numbers of signal events, keeping in mind that each analysis selects a subset of events with different properties, and hence different backgrounds, both with respect to size and composition.

In this work we will pursue two strategies: we begin with a more conventional search modelled loosely on the jet- $p_{T}$-based disappearing charged track analysis carried out by the ATLAS collaboration at $8 \mathrm{TeV}[28]$ with rescaled cuts. ${ }^{5}$ Our second approach is more speculative, employing a track-based analysis which will assume the successful use of some combination of charged-track momentum, and perhaps also $d E / d x$, to select potentially interesting events in a kinematic regime where they are not swamped by Standard Model

\footnotetext{
${ }^{4}$ Although the above distributions were obtained using the simplified model detailed in section 2 above, we expect their salient features to be mostly dependent on kinematics, and hence relatively insensitive to the particulars of the model used.

${ }^{5}$ The CMS analysis [29] has a higher mass reach, but less sensitivity at small lifetimes.
} 


\begin{tabular}{|ccc|ccc|}
\hline & \multicolumn{2}{c|}{ LHC14-HL } & \multicolumn{3}{c|}{ FCC-hh } \\
\cline { 2 - 6 } & Conventional & TB Central & Conventional & TB Central & TB Forward \\
\hline lepton veto & $\checkmark$ & $\checkmark$ & $\checkmark$ & $\checkmark$ & $\checkmark$ \\
$p_{T, j_{1}}(\mathrm{GeV})$ & 150 & & 1000 & & \\
$\mathbb{E}_{T}(\mathrm{GeV})$ & 150 & & 1000 & & \\
$\Delta \phi_{\min }^{j, \underline{t}_{T}}$ & 1.5 & & 1.5 & & \\
$p_{T, \operatorname{tr}}(\mathrm{GeV})$ & 350 & 1000 & 1000 & 3000 & \\
$p_{\operatorname{tr}}(\mathrm{GeV})$ & & & & & 8000 \\
$\left|\eta_{\operatorname{tr}}\right|$ & $(0.1,2)$ & $(0.1,2)$ & $(0,2)$ & $(0,2)$ & $(2,4)$ \\
$l_{\text {tr }}(\mathrm{cm})$ & $r=(10,65)$ & $r=(10,65)$ & $r=(10,65)$ & $r=(10,65)$ & $z=(45,70)$ \\
\hline
\end{tabular}

Table 1. Selection criteria for the conventional and track-based (TB) analyses presented in this paper, with $p_{T, \text { tr }}$ and $p_{\text {tr }}$ referring to the transverse and total momentum, respectively, of the charged track with length $l_{\text {tr }}$. Unless otherwise noted all values given are minimum values for the quantity in question.

QCD backgrounds. ${ }^{6}$ The exact value of the momentum trigger is not crucial here, since the charged particles with largest momenta have a higher probability of surviving to large distances, but for the purposes of this analysis we assume $|p|_{\text {track }} \gtrsim$ a few $\mathrm{TeV}$ will suffice. In each case we will explore the effect that varying the detector setup has on the sensitivity of the analysis in question. The detailed event selection for each study is summarized in table 1.

The sensitivity in this search is controlled by three parameters, the mass of the decaying charged particle $m_{\chi}$, its nominal decay length, $c \tau$, and the cross section for production of the charged state. The event kinematics and analysis efficiencies are fixed by the first two, we will treat these as free parameters and scan over them. The cross section sets the overall normalisation, and hence the number of events and search significances, and is a fixed function of mass for a specific choice of quantum numbers and interactions for the dark sector particles. We will present our results at two different levels of specificity: at the most general level we remain agnostic about the origin and properties of the dark sector, and provide information on the production cross section necessary to produce a fixed number of events (corresponding to an estimated $5 \sigma$ significance) for each mass and decay length, for ease of recasting. We will then use the simplified model described in section 2 to set the production rate, and translate these results into projected sensitivities in the $\left(m_{\chi}, c \tau\right)$ plane. Throughout this work, simulations will be carried out using this simplified model, although as stated above, we expect the analysis efficiencies to be rather insensitive to the details thereof.

\subsection{Conventional analysis}

We model this analysis on the disappearing charged track search carried out by the ATLAS collaboration at $8 \mathrm{TeV}[28]$ with cuts roughly scaled to $100 \mathrm{TeV}$ centre-of-mass. We use the

\footnotetext{
${ }^{6}$ We thank Phil Harris and Maurizio Pierini for bringing to our attention the possibility of using $d E / d x$ as a discriminating variable.
} 
following pre-selection criteria:

- lepton veto;

- leading jet $p_{T}>1 \mathrm{TeV}$;

- $\mathbb{E}_{T}>1 \mathrm{TeV}$

- $\Delta \phi_{\min }^{j, E_{T}}>1.5$ to reduce the contribution from hard jets faking MET.

In addition, ATLAS selects isolated charged tracks that give at least 5 hits in the inner tracker layers, of which 3 are in the pixel tracker and 2 in the (double-sided) SCT, to facilitate a good track reconstruction. In order to satisfy this criterion a track must reach a transverse distance $r$ of $30 \mathrm{~cm}$, severely compromising the sensitivity to intermediatelifetime particles. Instead, we will allow the radius at which $100 \%$ efficiency is reached for a disappearing charged track to vary, and show how this affects the sensitivity for different masses and lifetimes of the charged state. The pseudorapidity range of the ATLAS analysis is limited by the extent of the Transition Radiation Tracker (TRT), which is used to veto long-lived tracks, but as we shall show later increasing the $\eta$ range has little effect on the sensitivity of this analysis. We will also comment on the variation of the sensitivity with changes in the leading jet $p_{T}$ and MET cuts.

Our charged track selection is as follows:

- $p_{T, \text { track }}>1 \mathrm{TeV}$;

- transverse track length $r_{\text {min }} \leq r_{\text {track }} \leq 65 \mathrm{~cm}$, with $r_{\text {min }}$ the minimum length necessary for a reliable track reconstruction; ${ }^{7}$

- $0<\left|\eta_{\text {track }}\right|<2$,

for tracks which are assumed to satisfy isolation criteria similar to those used in the ATLAS analysis, rescaled to the busier $100 \mathrm{TeV}$ environment [28], possibly supplemented with a veto on large calorimeter deposits close to the extrapolated path of the charged particle leaving the track. We show in figure 4 the charged track distribution with transverse distance from the beamline $r$. In the left panel (a), we bin the distribution in $\eta$ for $m_{\chi}=1.1 \mathrm{TeV}$ and $p_{T}\left(j_{1}\right), \mathbb{E}_{T} \geq 1 \mathrm{TeV}$, while in the right panel (b) we integrate the total number of charged tracks over $|\eta| \leq 2$, for three different masses, and two different $p_{T}, \mathbb{E}_{T}, p_{T, \text { tr }}$ cuts. We take a nominal decay length of $6.6 \mathrm{~mm}$, which corresponds to the proper lifetime of the charged component of $1.1 \mathrm{TeV}$ pure-Higgsino state, for illustration. The sensitivity to charged tracks in the current ATLAS search begins at $r=30 \mathrm{~cm}$, shaded in cyan in the left panel. It is evident from the plots that $\mathrm{TeV}$-scale charged states with sub-cm $c \tau$ cannot make it out to large enough transverse distances in order to be covered by existing searches. Were it instead possible to achieve $100 \%$ track reconstruction efficiency within $10 \mathrm{~cm}$ of the beamline, one could have $\mathcal{O}(10)$ events for weak-doublet masses comfortably up to $1 \mathrm{TeV}$.

\footnotetext{
${ }^{7} \mathrm{We}$ also assume the presence of a tracker at $65 \mathrm{~cm}$ that fulfils the role of the TRT.
} 


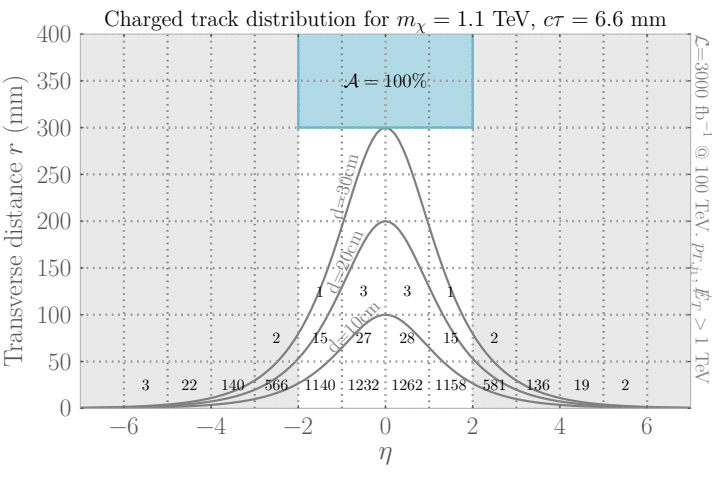

(a)

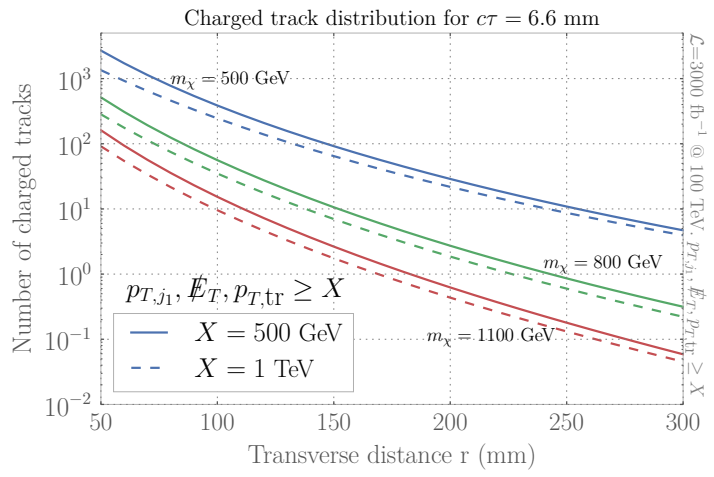

(b)

Figure 4. Distribution of number of charged tracks for weak-doublet fermion $\chi$ pair-produced at FCC-hh, as a function of $r(\mathrm{~mm})$, (a) binned in $\eta$ and rounded down to the nearest integer, for $m_{\chi}=1.1 \mathrm{TeV}$ and $p_{T}\left(j_{1}\right), \mathbb{E}_{T}, p_{T, \mathrm{tr}}>1 \mathrm{TeV}$ and (b) integrated over $|\eta| \leq 2$, for various masses and $p_{T}\left(j_{1}\right), \mathbb{E}_{T}>X$. The lifetime is chosen equal to that of the corresponding pure-Higgsino state in the MSSM, $c \tau=6.6 \mathrm{~mm}$. The existing ATLAS search only has sensitivity for tracks that reach $r=30 \mathrm{~cm}$, shown as a cyan shaded region in the left panel (at CMS this number is slightly larger).

Improving the track resolution at small $r$ does not seem outside the realm of possibility, ${ }^{8}$ and one could envision various alternative tracker arrangements that might achieve this. Putting additional pixel barrel layers in-between existing ones might suffice; as might angling the pixel elements with respect to the beamline, as in the ATLAS Alpine upgrade proposal, which could yield multiple hits from a single layer. Since it is difficult to accurately assess the cost and relative feasibility of such ideas, we make no further comment on the matter, and simply assume in what follows that a reliable charged track resolution can be achieved within a radial distance of $10 \mathrm{~cm}$. We then show in the left panel of figure 5 contours of the effective cross section required to obtain 10 charged track events at $r=10 \mathrm{~cm}$, as a function of $m_{\chi}$ and $c \tau$. The 'effective' nature of the cross section stems from the fact that pair-production of a weak multiplet includes sub-processes with different numbers of charged states, each of which has a different cross section, and is defined by $\sigma_{\text {eff }} \mathcal{L}=N_{\chi^{ \pm}}$, the number of charged states produced. This plot can then be used to estimate the sensitivity for any scenario with direct pair-production of a short-lived charged particle. ${ }^{9}$ We see for instance, that obtaining 10 charged tracks at $r=10 \mathrm{~cm}$ for TeV-scale charged particles and $c \tau$ between 5 and $10 \mathrm{~mm}$ would require a cross section of $\sim 100 \mathrm{fb}$, in the correct ball-park for weak production.

We also show in figure 5(b) contours of the total number of charged tracks as function of $c \tau$, assuming a 100\%-efficient disappearing track selection at $r=10 \mathrm{~cm}$. This is normalized

\footnotetext{
${ }^{8}$ Indeed the compressed Wino limits presented by ATLAS subsequent to the publication of this article [40] were obtained using 12 -cm tracks with no changes to detector hardware necessary.

${ }^{9}$ Caution must be exercised in applying the results to charged particles produced in cascade decays, whose boost spectrum is instead set by the mass differences between the parent and daughter particles, and could be unlike that of a particle pair-produced at threshold.
} 


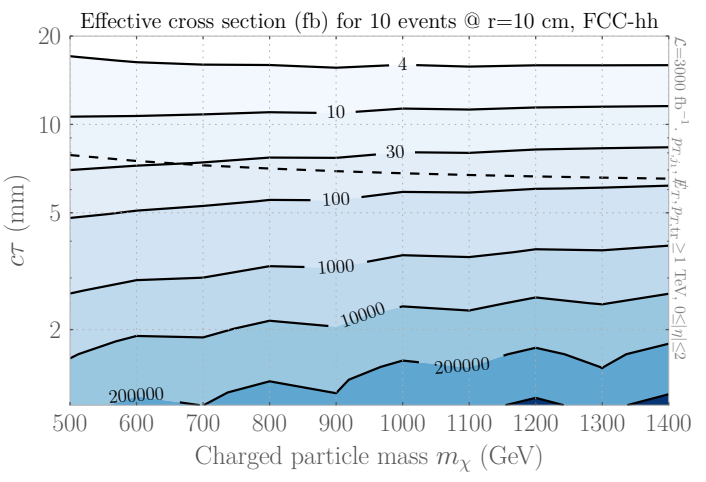

(a)

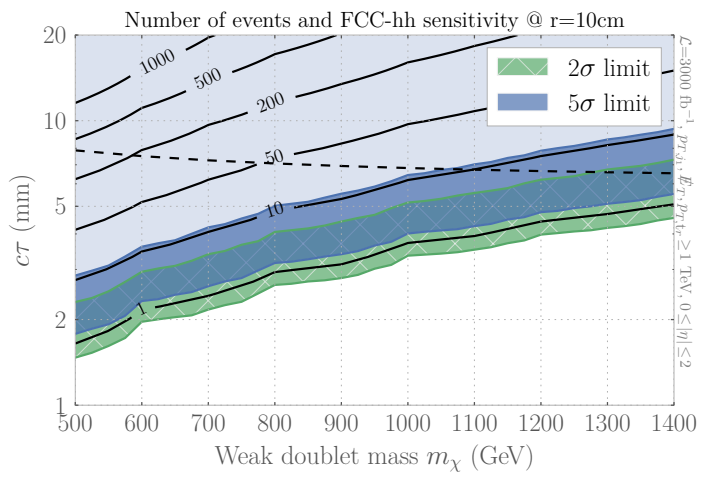

(b)

Figure 5. Results of conventional analysis: (a) Effective charged particle production cross section (definition in text) required in order to obtain 10 disappearing charged track events in conventional analysis at $r=10 \mathrm{~cm}$, and (b) number of disappearing charged tracks and sensitivity, normalized to the NLO pair-production cross section of a weak-doublet fermion with Dirac mass $m_{\chi}$ and nominal decay length $c \tau$. The plots are for a $p p$ collider at $\sqrt{s}=100 \mathrm{TeV}$ with $3000 \mathrm{fb}^{-1}$ of integrated luminosity. The $c \tau$ corresponding to a pure-Higgsino state is shown as a dotted line. Superimposed onto the right panel (grey shaded region) is the FCC-hh sensitivity in this channel for a $50 \%$ background systematic, with the estimated uncertainties in the $5 \sigma(2 \sigma)$ contours shaded in blue (green).

to the NLO production cross section for a weak-doublet fermion with Dirac mass $m_{\chi}$, at a $100 \mathrm{TeV} p p$ collider with $3000 \mathrm{fb}^{-1}$ of integrated luminosity. In both cases the $c \tau$ for a pure-Higgsino state is shown as a dotted line.

Converting a number of tracks to a discovery/exclusion significance requires some knowledge of the size of SM backgrounds to this process. There are essentially no real backgrounds satisfying the analysis criteria. ${ }^{10}$ Fake backgrounds consist of interacting hadron tracks, leptons failing identification criteria at low track $p_{T}$, and tracks with mismeasured $p_{T}$ due to "a high density of silicon hits, hadronic interactions and scattering" [28] at large track $p_{T}$. These fakes are not well-described by Monte Carlo simulations at the LHC at $8 \mathrm{TeV}$ centre-of-mass. Instead, their $p_{T}$ spectra are fit to data in specific 'control' regions and subtracted, rendering their extrapolation to $100 \mathrm{TeV}$ rather difficult. In addition their composition and spectra are characteristic of the particular detector in which they are measured (ATLAS in this instance), and a naive extrapolation to a hypothetical detector for a $100 \mathrm{TeV}$ hadron machine, with unknown properties, would be crude at best. Nevertheless we will make some attempt to do this. First, we assume that the fake

\footnotetext{
${ }^{10}$ In principle a very boosted charged B-hadron could give rise to a disappearing track if it passed most of its momentum to its neutral decay products. While the probability of a parent with $p_{T}>1 \mathrm{TeV}$ to decay to a charged state with momentum below the detection threshold is already vanishingly small, the neutral decay products would still leave large energy deposits in the calorimeters so even those events could be removed efficiently with suitable isolation cuts. What remains are events where the hadron decays to a charged state and missing energy (i.e. neutrinos), which is suppressed in the SM due to the absence of flavour-changing neutral currents.
} 
backgrounds at FCC-hh have a similar composition and are again dominated at high track $p_{T}$ by the mismeasured hadronic tracks satisfying the ATLAS $8 \mathrm{TeV}$ disappearing track selection, which is fitted using a control region consisting of disappearing tracks with large impact parameter, see [28] for details. Since these likely originate in high- $p_{T}$ collisional backgrounds, which scale like the falling PDFs, we assume the hadronic fakes satisfying our modified selection criteria retain the same scaling with track $p_{T}$ as the original $\left(p_{T, \text { track }}^{-a}\right.$ with $a=1.78 \pm 0.5$ ), with a floating overall normalization that parametrizes our uncertainty. This normalization constant can be estimated using the scaling of some chosen process with centre-of-mass energy. Previous works [5, 6] used Standard Model $(Z \rightarrow \nu \nu)$ plus jets, the rate for this process scales with the product of the quark and gluon PDFs. In order to be maximally conservative, we will also show the outcome using the scaling of SM multijets, with large fake MET. This is glue-glue-initiated, and hence grows faster with energy. We estimate the significance using the usual gaussian expression,

$$
\text { Significance }=\frac{S}{\sqrt{B+\lambda^{2} B^{2}}}
$$

for $S$ signal events and $B$ background events, and background systematic uncertainty $\lambda=50 \%$, using the difference between the two methods of scaling the backgrounds as a measure of our total background uncertainty. We superimpose the resulting discovery $(5 \sigma)$ and exclusion $(2 \sigma)$ bands on to the event count contours in figure $5(\mathrm{~b})$. The grey shaded region shows the extent of the FCC sensitivity, with the width of the coloured bands representing our estimated uncertainty in the significance contours. We see that the FCC with modified tracking can comfortably cover $c \tau$ down to about $7 \mathrm{~mm}$ for $m_{\chi} \sim 1 \mathrm{TeV}$. The extent of the sensitivity at larger masses depends on which estimate of the backgrounds is closer to reality, with our optimistic projection extending discovery reach to $m_{\chi}=1.4 \mathrm{TeV}$.

\subsection{Track-based (TB) analysis}

At the beginning of this section we argued that cuts in recoil $p_{T}$ and MET work to our disadvantage since they exclude events containing particles with the largest boost, which travel furthest from the interaction point. In this re-designed analysis we will dispense with recoil $p_{T}$ and related quantities, both for the purposes of triggering and analysis cuts. Instead we will cut directly on the measured track momentum, which is a proxy for the charged particle's boost, and hence the average lab-frame track length, for a fixed $c \tau$. We will assume that a hard enough (multi- $\mathrm{TeV}$ ) trigger requirement on this quantity, perhaps in association with a $d E / d x$-based selection, will reduce data storage rates and backgrounds to manageable levels. However, since it is impossible for us to make a reliable estimate of the size of fake backgrounds satisfying these modified disappearing track criteria, we will simply compare the sensitivity of our track-based analysis to the more conventional one at the level of the number of charged tracks.

We perform our track-based analysis in two non-overlapping signal regions. The central region $0 \leq|\eta| \leq 2$ will contain the bulk of the charged tracks. In addition, we define a forward region $2 \leq|\eta| \leq \eta_{\max }$ for some $\eta_{\max }$ to be specified later, which contains fewer but highly-boosted charged states, as illustrated in figure 3(c). The former will share part 


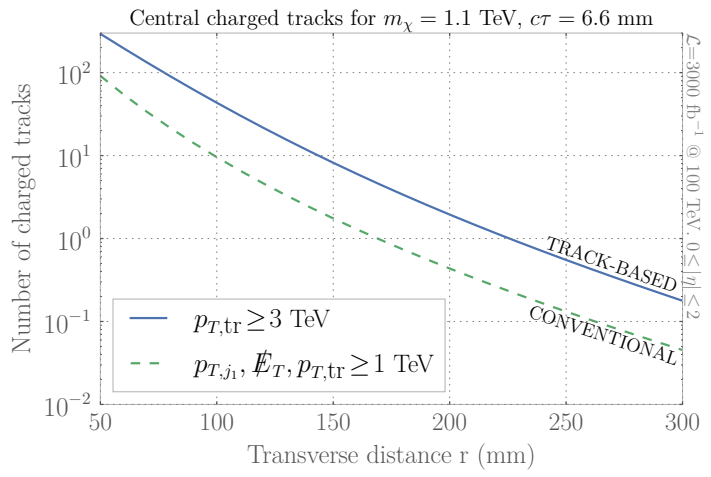

(a)

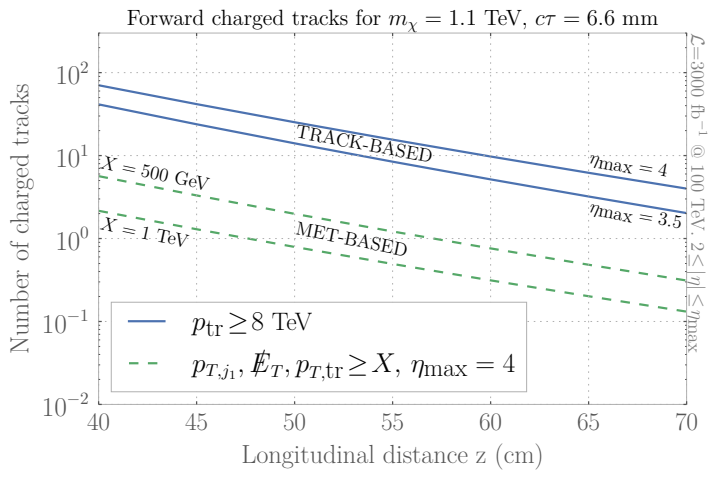

(b)

Figure 6. Number of charged tracks in track-based (TB) analysis for reference values $m_{\chi}=$ $1.1 \mathrm{TeV}, c \tau=6.6 \mathrm{~mm}$, in (a) central region as a function of transverse distance $r$ from the beamline, with the number of tracks in the conventional analysis shown for comparison, and (b) forward region as a function of the longitudinal distance $z$ from the interaction point, for two different values of the pseudorapidity extent. We also show the number of charged tracks available in the forward direction using standard jet $p_{T}$ and MET cuts.

of the disappearing charged-track selection criteria of the conventional analysis described above, but none of the event-selection, which relied on hard additional radiation. In the central track selection we require:

- $p_{T, \operatorname{tr}} \geq 3 \mathrm{TeV}$

- $0 \leq|\eta| \leq 2$;

- $10 \mathrm{~cm} \leq r_{\mathrm{tr}} \leq 65 \mathrm{~cm}$.

The charged track distribution as a function of $r$, for $m_{\chi}=1.1 \mathrm{TeV}, c \tau=6.6 \mathrm{~mm}$ is shown in figure 6(a), as is the analogous distribution for the conventional analysis. We see that dropping the hard radiation requirement increases the number of charged track events by a factor of 4 at a transverse distance of $10 \mathrm{~cm}$, yielding more than 40 events for our reference value.

We further defining a track-based forward region as follows:

- $p_{\mathrm{tr}} \geq 8 \mathrm{TeV}$

- $2 \leq|\eta| \leq 4$.

Now we study the charged track distribution as a function of longitudinal distance $z$ from the interaction point, for two different values of $\eta_{\max }$ (see figure $6(\mathrm{~b})$ ), with the smaller value giving a factor of 2 decrease in charged track yield. By contrast, there are an order of magnitude fewer tracks available if the traditional $p_{T, \mathrm{j}} /$ MET-based cuts are maintained.

A complete definition of the forward signal region requires a concrete proposal for a tracker that can successfully identify disappearing charged tracks in the forward direction. 


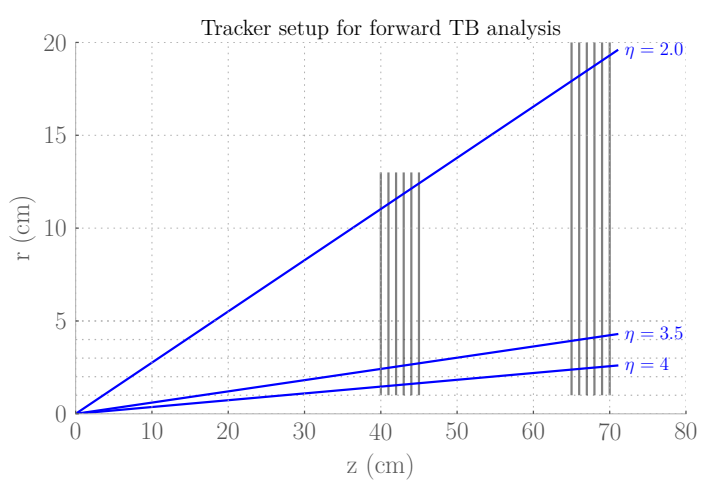

Figure 7. Sketch of the proposed forward tracker setup, consisting of two endcaps, each $5 \mathrm{~cm}$ wide, located at $z=40$ and $65 \mathrm{~cm}$, providing hermetic coverage of the forward region $2 \leq|\eta| \leq 4$, for example. We assume any track reaching the far side of the near endcap has yielded sufficient tracker hits to allow a reliable track reconstruction, and, veto on tracks traversing the far endcap.

We have in mind a setup similar to figure 7 with four tracking endcaps (two at each end) providing hermetic coverage of the forward region $2 \leq|\eta| \leq 4$, the far sides of which we take to be located at $z=45$ and 70. Although the endcaps, as illustrated, have a width of $5 \mathrm{~cm}$, the precise value is irrelevant provided we can assume any track reaching the far side of the near endcap has left enough tracker hits to give a reliable track reconstruction. We also veto any charged track that traverses the far endcap, which then plays the role of the TRT in the $8 \mathrm{TeV}$ ATLAS analysis, allowing us to exclude long-lived charged particles. ${ }^{11}$ We can then add to the TB forward track-selection criteria the following requirement on track length: $45 \mathrm{~cm} \leq z_{\text {tr }} \leq 70 \mathrm{~cm}$.

We display in figure 8 (a) and (b) contours of the total number of tracks in the TB central and forward analyses respectively, as a function of the weak-doublet mass $m_{\chi}$ and nominal decay length $c \tau$. As before the dashed line corresponds to the $c \tau$ for a pureHiggsino state. Each of these track-based searches gives a four-fold increase in the number of signal events relative to the conventional analysis.

\subsection{LHC14-HL sensitivity}

We can further compare these results to those obtainable at a high-luminosity $14-\mathrm{TeV}$ run of the LHC, with $3000 \mathrm{fb}^{-1}$ integrated luminosity, assuming similar modifications are possible via detector upgrades. Our selection criteria for the conventional analysis, now scaled to $14 \mathrm{TeV}$ centre-of-mass, are:

- $p_{T, j_{1}}, \mathbb{E}_{T} \geq 150 \mathrm{GeV}$;

- $p_{T, \mathrm{tr}} \geq 350 \mathrm{GeV}$;

with the requirement on the charged track geometry unchanged from section 3.1 above. For the central and forward track-based analyses we simply modify the track momentum cuts

\footnotetext{
${ }^{11}$ Note that coverage to $\eta=4$ for the chosen endcap width mandates the presence of tracker material within $r=1.5 \mathrm{~cm}$ of the beamline at $z=40 \mathrm{~cm}$. This requirement can be relaxed by reducing the pseudorapidity coverage or by moving the near endcap further from the interaction point, at the cost of decreasing the sensitivity.
} 


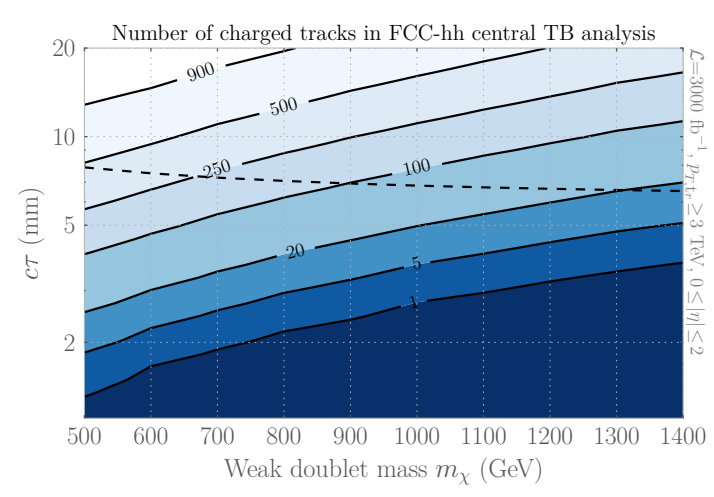

(a)

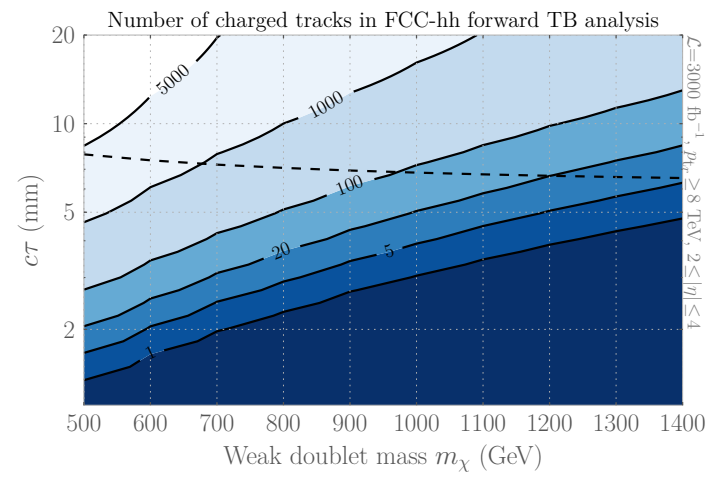

(b)

Figure 8. Number of charged tracks in (a) central TB analysis (b) forward TB analysis, normalized to the NLO cross section of a pair-produced weak-doublet fermion with Dirac mass $m_{\chi}$ and nominal decay length $c \tau$. The $c \tau$ corresponding to a pure-Higgsino state is shown as a dashed line.

of the FCC-hh version, to $p_{T, \mathrm{tr}} \geq 1 \mathrm{TeV}$ and $p_{\mathrm{tr}} \geq 1.5 \mathrm{TeV}$, respectively. Our results, normalized to the NLO cross section at the LHC14 from figure 2(a), are shown in figure 9 . The backgrounds for the conventional analysis, panel (a), were estimated as before, with the ATLAS $8 \mathrm{TeV}$ limits on a pure Wino state in the same channel are shaded in yellow. Recall that the production cross section of the Wino is larger than that of the Higgsino by a weak group Casimir factor. We also show our LHC14-HL projection with unchanged tracking capability in the same plot for reference. We see that increasing the tracker granularity below $r=10 \mathrm{~cm}$ as proposed above can result in a factor of 3 increase in sensitivity at LHC14-HL, extending the discovery reach down to $c \tau \sim 20 \mathrm{~cm}$ for $m_{\chi}=600 \mathrm{GeV}$. In this lower-energy environment the central TB analysis (figure 9(c)) still gives a two-fold enhancement to the number of signal events. The forward TB analysis (panel (d)) however is not as effective due to the smaller overall boost, improving the charged-track yield by less than $30 \%$.

A quantitative assessment of the negative impact on the TB signal region of radiatedjet $p_{T}$ cuts can be found in appendix B. Note that all our analyses above are simple cut-and-counts, un-optimized for the range of mass scales they are applied to. As a result our conclusions can be thought of as rather conservative, with a dedicated optimization expected to yield improved sensitivity over the entire mass range.

\section{Conclusions}

One reason why we have seen no evidence of New Physics at the LHC could be that the lowest-lying new states have compressed spectra. If these new states form part of a dark sector, with splittings so small that the SM by-products of decays within the sector become too soft to be detected, we have few handles to use in searching for such scenarios at hadron colliders. If the compressed dark sector contains a charged component, the disappearing track of this charged particle can be used in searches. Such disappearing tracks will satisfy modified tracking criteria, and building in sensitivity to this type of 


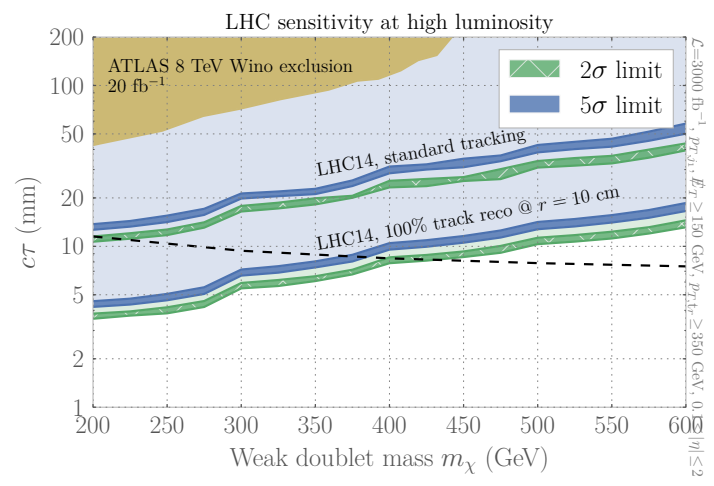

(a)

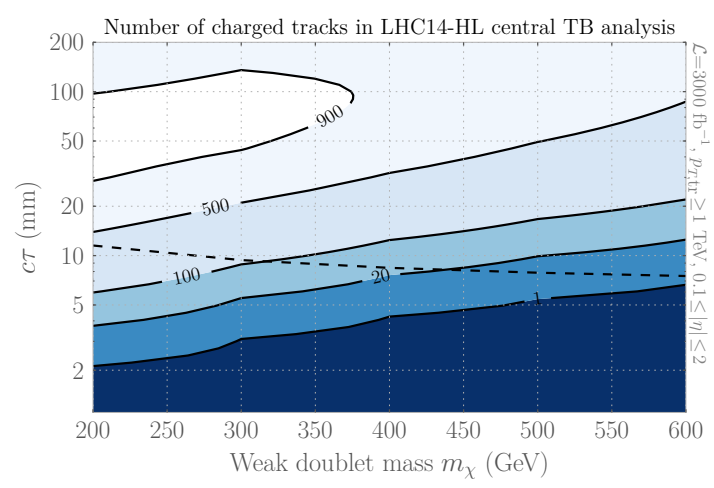

(c)

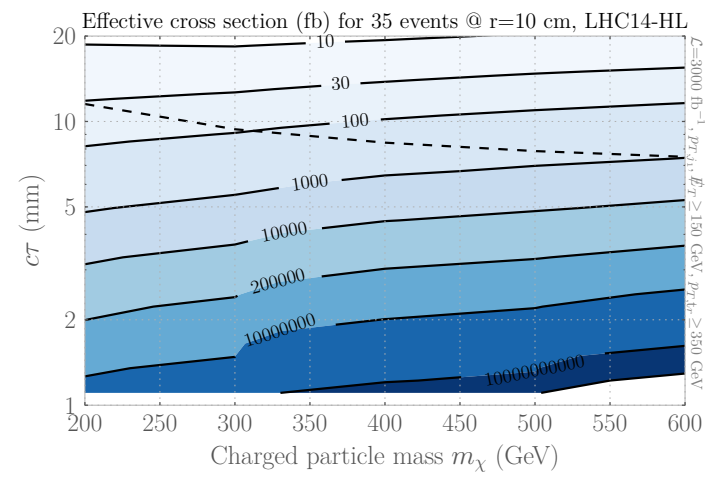

(b)

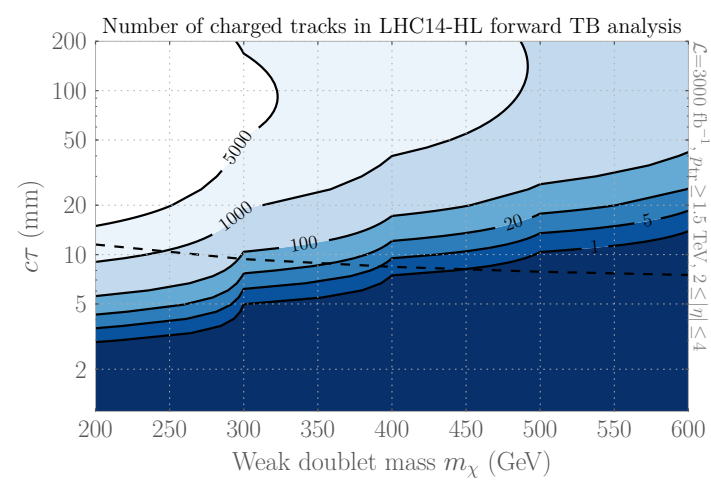

(d)

Figure 9. Reach for a high-luminosity run of LHC14, with $\mathcal{L}=3000 \mathrm{fb}^{-1}$ integrated luminosity in (a) conventional analysis with and without (specified) improvements in tracker granularity close to the beamline. We assume $50 \%$ background systematics, with estimated uncertainties (details in text) in the $5 \sigma$ exclusion ( $2 \sigma$ discovery) contours shaded in blue (green). The yellow shaded region corresponds to the ATLAS $8 \mathrm{TeV}$ limit for pure Wino states. (b) Effective cross section (defined in text) required for 35 charged-track events in conventional analysis at LHC14-HL, corresponding to a (conservative) $5 \sigma$ discovery reach. (c) Number of disappearing charged tracks satisfying selection criteria in central TB analysis. (d) Number of disappearing charged tracks satisfying selection criteria in forward TB analysis. The dashed line corresponds to the nominal decay length for pure-Higgsino states.

exotic signature at future detectors will maximize our chances of closing the net for New Physics at future colliders.

In this work we focused on extending the sensitivity of a future $100 \mathrm{TeV}$ proton-proton collider to disappearing charged tracks with sub-cm $c \tau$, by modifying detector specifications. While our motivation partly arises from covering $\mathrm{TeV}$-scale masses (inspired by pure-Higgsino dark matter) our results are very general and apply to a variety of dark sector models.

We first explored the effect of small modifications to the tracker setup on the outcome of conventional searches. While current searches are insensitive to tracks that fall shy of $30 \mathrm{~cm}$ in the transverse direction, a reliable reconstruction of charged tracks within $10 \mathrm{~cm}$ 
of the beampipe would increase the reach in $c \tau$ by a factor of 3 at the high-luminosity run of LHC14, and a further factor of 5 at FCC-hh, effectively testing nominal decay lengths of a few millimetres.

Furthermore our simulations showed that employing hard cuts on the transverse momentum of SM radiation (and the related quantity $\mathbb{E}_{T}$ ) removes a significant fraction of the signal events with the longest lab-frame decay lengths. In order to seize these highlyboosted particles, we proposed selecting events by requesting large overall momentum of a charged track, thus removing the need for additional radiation to discriminate the event. We explored two different setups, relying on the use of a) the central region, $0<|\eta|<2$, with modifications in tracking resolution as per our 'conventional' analysis and b) the forward region, $2<|\eta|<4$ with additional tracking endcaps and a charged particle boost factor $\gamma \beta$ about twice that of the central region. As both regions are expected to be subject to different backgrounds, we refrain from performing a sensitivity estimation, but rather design our cuts so as to obtain some modest number of signal events. Taking the conventional $p_{T}$ and $\mathbb{E}_{T}$-based analysis as a baseline, our new track-based search gives 5 times more observable signal events in each of the central and forward regions, offering tremendous potential for improvement. A similar track-based search at LHC-HL would increase the number of events in the central region by a factor of 2 , while the forward region suffers from the lower available boost and is therefore less promising. While the potential increase in sensitivity is not as dramatic as at the FCC-hh, studying the feasibility of implementing track-based triggers at the LHC-HL still seems worthwhile.

An alternative way of implementing the central TB analysis is as follows: imagine defining track-MET as the missing transverse energy computed using on tracks in the inner $10 \mathrm{~cm}$ of the tracker. By construction, signal events will have track-MET of the order of the chargino $p_{T}$, while at the calorimeter level the 'calo-MET' is set by the $p_{T}$ of the leading jet. Background events by contrast, will not have a large discrepancy between track- and calo-MET, and hence the ratio of these two variables stands as a powerful discriminator between signal and background. While here this merely amounts to a rephrasing of the performed analysis, this ratio could also be employed to isolate more complex signals like Emerging Jets [41], where a large number of tracks may appear or disappear.

Here we have seen that track-based searches with reduced dependence on inclusive kinematic variables are a promising direction for new physics searches, offering significant improvements in the experimental reach for larger masses and shorter lifetimes. Combining the search strategies explored in this paper will further improve our sensitivity to charged states with picosecond lifetimes, allowing us to definitively discover, or exclude, a pureHiggsino thermal relic at $100 \mathrm{TeV}$.

\section{Acknowledgments}

We would like to thank Benjamin Fuks, Roberto Franceschini, Frank Krauss, Gavin Salam, Michele Selvaggi and Jure Zupan for useful discussions, as well as the Galileo Galilei Institute for Theoretical Physics, the Mainz Institute of Theoretical Physics (MITP) and the Theory group at CERN for their warm hospitality during the completion of this work. RM 
was partially supported by ERC grant 614577 "HICCUP" High Impact Cross section Calculations for Ultimate Precision, and the Swiss National Science Foundation under MHV grant 171330. P.S is supported by the DFG Cluster of Excellence PRISMA (EXC 1098).

\section{A Decays and lifetimes}

For mass splittings that correspond to the lifetime range under consideration, the charged state in the doublet will decay via off-shell $\mathrm{W}$ bosons into the lighter neutral state, accompanied predominantly by either a single soft pion or a lepton-neutrino pair. The partial widths for the relevant channels were computed in [30], and are given below for convenience. They agree with the expressions found in refs. [42, 43] for the Higgsino in the pseudo-Dirac limit, where the Majorana splitting between the two neutralinos is taken much smaller than the chargino-neutralino splitting $\Delta^{+}$, and the decay widths into both neutralinos are summed.

The partial width into the neutral component plus two SM fermions, $f$ and $\bar{f}^{\prime}$, with $m_{f^{\prime}} \sim 0$ is:

$$
\Gamma\left(\chi^{-} \rightarrow \chi_{0} f \bar{f}^{\prime}\right)=\frac{2 N_{C} G_{F}^{2}\left(\Delta_{+}\right)^{5}}{15 \pi^{3}} \sqrt{1-r_{f}^{2}} \mathcal{P}\left(r_{f}\right)
$$

where $N_{C}$ is the number of fermion colours, $r_{f}=m_{f} / \Delta^{+}$and

$$
\mathcal{P}\left(r_{f}\right)=1-\frac{9}{2} r_{f}^{2}-4 r_{f}^{4}+\frac{15 r_{f}^{4}}{2 \sqrt{1-r_{f}^{2}}} \tanh ^{-1} \sqrt{1-r_{f}^{2}} .
$$

The partial width to a charged pion plus neutral component is given by:

$$
\Gamma\left(\chi^{-} \rightarrow \chi^{0} \pi^{-}\right)=\frac{2 G_{F}^{2} f_{\pi}^{2} \cos ^{2} \theta_{C}}{\pi} \Delta_{+}^{3}\left(1-\frac{r_{\pi}^{2}}{2}\right) \sqrt{1-r_{\pi}^{2}}
$$

where $f_{\pi} \approx 91.9 \mathrm{GeV}$ is the pion decay constant, $\theta_{C}$ is the Cabbibo angle and $r_{\pi}=m_{\pi} / \Delta_{+}$.

Expressions for subdominant modes including the decays into two and three pions can be found in ref. [42]. They are not particularly illuminating but for completeness we have included them in our numerical analysis. The overall impact of these channels is small except for the region [0.7-2.5] GeV where the two-pion channel contributes $\mathcal{O}(10 \%)$ to the branching ratio. We show in the left panel of figure 10 the branching ratios of the charged state $\chi^{+}$into $\chi^{0}+X$ for $m_{\chi}=1.1 \mathrm{TeV}$, while in the right panel we show the corresponding nominal decay length $c \tau$, with the blue shaded region corresponding to the viable range of pure-Higgsino parameter space. Both the branching fractions and decay lengths are highly insensitive to the value of the Dirac mass in the limit $m_{\chi} \gg \Delta_{+}$, but are instead very sensitive to the splitting, increasing like $\sim \Delta_{+}^{3}$ in the entire region of interest. For values of the splitting given by the one-loop electroweak contribution, the branching fraction to a single-pion final state is $97 \%$. Due to the small decay length of the chargino we have restricted ourselves to $\Delta_{+}<1.5 \mathrm{GeV}$. For this mass range we have explicitly checked that transitioning to a parton framework to compute this decays gives smaller values than the hadronic approach. Moving to heavier masses requires to perform a matching between both frameworks (see reference [42]). 


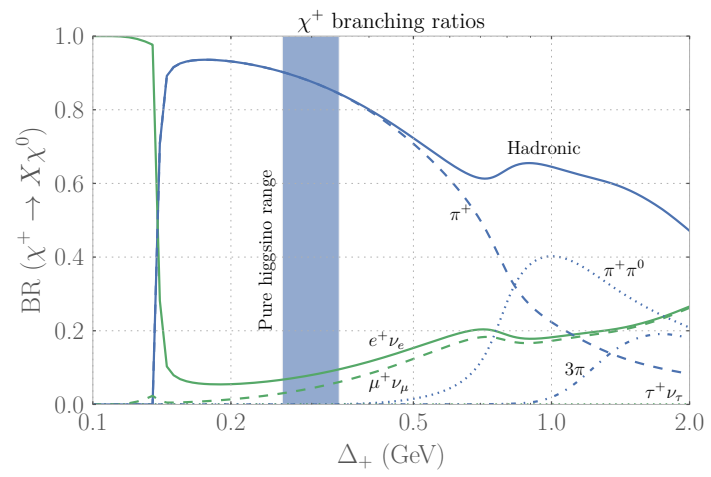

(a)

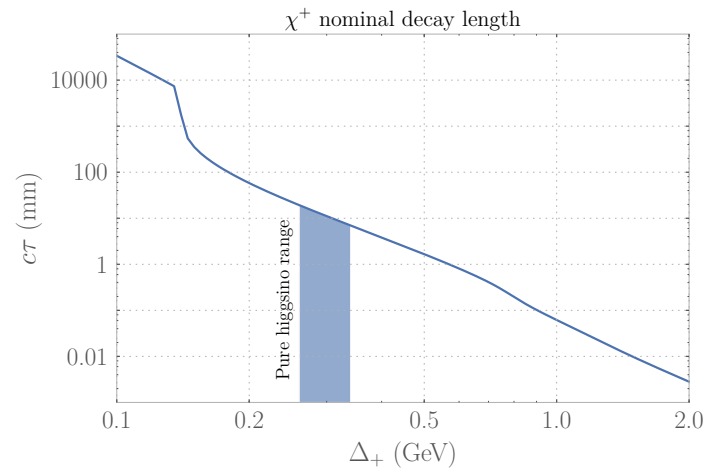

(b)

Figure 10. Branching ratios (left) and nominal decay length (right), for the charged component of an electroweak doublet with hypercharge $1 / 2$ and Dirac mass $m_{\chi}=1.1 \mathrm{TeV}$, as a function of $\Delta_{+}$, the splitting between the charged and neutral states. Note that the partial widths are highly insensitive to the overall mass scale.

\section{B Impact of transverse cuts on forward track-based analysis}

While one of the main messages of this work was to propose the replacement of the transverse variables by track-related ones, it is still important to quantify the effect of performing transverse cuts since they could help keeping backgrounds under control. We thus quantify here the degradation of the signal in the TB searches due to transverse cuts by counting the number of charged tracks satisfying our selection criteria as a function of a soft cut on the $p_{T}$ of the leading jet, $p_{T, j 1} \geq X$. The results for FCC-hh and LHC14-HL are presented in figure 11. We see that even a very soft cut of $p_{T, j_{1}} \geq 100 \mathrm{GeV}$ at the FCC-hh decreases by a factor of two the number of signal events available in the forward region, and keeps about $75 \%$ of the events in the central region. For the LHC14 we observe a similar behaviour for $p_{T, j_{1}} \geq 50 \mathrm{GeV}$. As a result, one must be cautious when using radiated jet $p_{T}$ in $\mathrm{TB}$ analyses, as even rather soft cuts sizably reduce the number of signal events. 


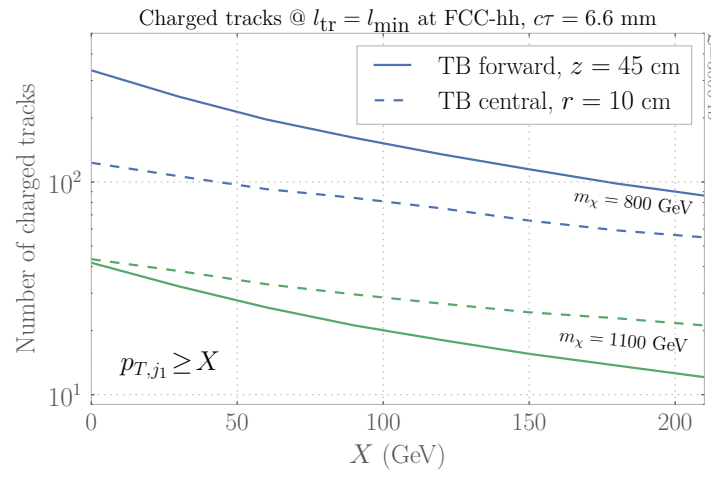

(a)

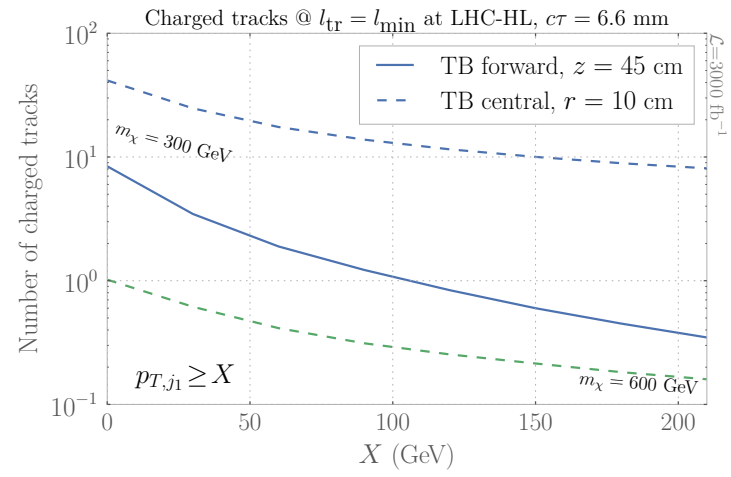

(b)

Figure 11. (a) Number of charged tracks in track-based analyses, at $z=45 \mathrm{~cm}$ and $r=10$ $\mathrm{cm}$ in central region, as a function of a cut on the transverse momentum of the hardest recoil jet $p_{T, j_{1}}>X$. The total rate is normalized to the NLO production cross section for a weak doublet with mass $1.1 \mathrm{TeV}$ and $c \tau=6.6 \mathrm{~mm}$ at (a) a $100 \mathrm{TeV} p p$ collider and (b) a $14 \mathrm{TeV} p p$ collider, with $3000 \mathrm{fb}^{-1}$ of integrated luminosity.

Open Access. This article is distributed under the terms of the Creative Commons Attribution License (CC-BY 4.0), which permits any use, distribution and reproduction in any medium, provided the original author(s) and source are credited.

\section{References}

[1] M.J. Strassler and K.M. Zurek, Echoes of a hidden valley at hadron colliders, Phys. Lett. B 651 (2007) 374 [hep-ph/0604261] [INSPIRE].

[2] Z. Chacko, H.-S. Goh and R. Harnik, The twin Higgs: Natural electroweak breaking from mirror symmetry, Phys. Rev. Lett. 96 (2006) 231802 [hep-ph/0506256] [INSPIRE].

[3] G. Burdman, Z. Chacko, H.-S. Goh and R. Harnik, Folded supersymmetry and the LEP paradox, JHEP 02 (2007) 009 [hep-ph/0609152] [INSPIRE].

[4] J.P. Chou, D. Curtin and H.J. Lubatti, New Detectors to Explore the Lifetime Frontier, Phys. Lett. B 767 (2017) 29 [arXiv: 1606.06298] [InSPIRE].

[5] M. Low and L.-T. Wang, Neutralino dark matter at $14 \mathrm{TeV}$ and $100 \mathrm{TeV}$, JHEP 08 (2014) 161 [arXiv: 1404.0682] [INSPIRE].

[6] M. Cirelli, F. Sala and M. Taoso, Wino-like Minimal Dark Matter and future colliders, JHEP 10 (2014) 033 [Erratum ibid. 1501 (2015) 041] [arXiv: 1407.7058] [INSPIRE].

[7] J. Halverson, N. Orlofsky and A. Pierce, Vectorlike Leptons as the Tip of the Dark Matter Iceberg, Phys. Rev. D 90 (2014) 015002 [arXiv:1403.1592] [InSPIRE].

[8] S. Jung and H.-S. Lee, Constraining Higgsino Kink Tracks from Existing LHC Searches, Int. J. Mod. Phys. A 32 (2017) 1750070 [arXiv:1503.00414] [INSPIRE].

[9] B. Ostdiek, Constraining the minimal dark matter fiveplet with LHC searches, Phys. Rev. D 92 (2015) 055008 [arXiv: 1506.03445] [INSPIRE]. 
[10] V.V. Khoze, A.D. Plascencia and K. Sakurai, Simplified models of dark matter with a long-lived co-annihilation partner, JHEP 06 (2017) 041 [arXiv:1702.00750] [INSPIRE].

[11] G.F. Giudice, T. Han, K. Wang and L.-T. Wang, Nearly Degenerate Gauginos and Dark Matter at the LHC, Phys. Rev. D 81 (2010) 115011 [arXiv:1004.4902] [INSPIRE].

[12] S. Gori, S. Jung and L.-T. Wang, Cornering electroweakinos at the LHC, JHEP 10 (2013) 191 [arXiv:1307.5952] [INSPIRE].

[13] C. Han, A. Kobakhidze, N. Liu, A. Saavedra, L. Wu and J.M. Yang, Probing Light Higgsinos in Natural SUSY from Monojet Signals at the LHC, JHEP 02 (2014) 049 [arXiv: 1310.4274] [INSPIRE].

[14] P. Schwaller and J. Zurita, Compressed electroweakino spectra at the LHC, JHEP 03 (2014) 060 [arXiv: 1312.7350] [INSPIRE].

[15] H. Baer, A. Mustafayev and X. Tata, Monojets and mono-photons from light higgsino pair production at LHC14, Phys. Rev. D 89 (2014) 055007 [arXiv:1401.1162] [INSPIRE].

[16] Z. Han, G.D. Kribs, A. Martin and A. Menon, Hunting quasidegenerate Higgsinos, Phys. Rev. D 89 (2014) 075007 [arXiv:1401.1235] [INSPIRE].

[17] J. Bramante, A. Delgado, F. Elahi, A. Martin and B. Ostdiek, Catching sparks from well-forged neutralinos, Phys. Rev. D 90 (2014) 095008 [arXiv: 1408.6530] [InSPIRE].

[18] C. Han, L. Wu, J.M. Yang, M. Zhang and Y. Zhang, New approach for detecting a compressed bino/wino at the LHC, Phys. Rev. D 91 (2015) 055030 [arXiv:1409.4533] [INSPIRE].

[19] H. Baer, A. Mustafayev and X. Tata, Monojet plus soft dilepton signal from light higgsino pair production at LHC14, Phys. Rev. D 90 (2014) 115007 [arXiv:1409.7058] [INSPIRE].

[20] S. Gori, S. Jung, L.-T. Wang and J.D. Wells, Prospects for Electroweakino Discovery at a $100 \mathrm{TeV}$ Hadron Collider, JHEP 12 (2014) 108 [arXiv:1410.6287] [INSPIRE].

[21] T. Liu, L. Wang and J.M. Yang, Pseudo-goldstino and electroweakinos via VBF processes at LHC, JHEP 02 (2015) 177 [arXiv:1411.6105] [INSPIRE].

[22] J. Bramante et al., Relic Neutralino Surface at a 100 TeV Collider, Phys. Rev. D 91 (2015) 054015 [arXiv: 1412.4789] [INSPIRE].

[23] C. Han, D. Kim, S. Munir and M. Park, Accessing the core of naturalness, nearly degenerate higgsinos, at the LHC, JHEP 04 (2015) 132 [arXiv:1502.03734] [INSPIRE].

[24] D. Barducci, A. Belyaev, A.K.M. Bharucha, W. Porod and V. Sanz, Uncovering Natural Supersymmetry via the interplay between the LHC and Direct Dark Matter Detection, JHEP 07 (2015) 066 [arXiv: 1504.02472] [INSPIRE].

[25] M. Badziak, A. Delgado, M. Olechowski, S. Pokorski and K. Sakurai, Detecting underabundant neutralinos, JHEP 11 (2015) 053 [arXiv: 1506.07177] [INSPIRE].

[26] A. Ismail, E. Izaguirre and B. Shuve, Illuminating New Electroweak States at Hadron Colliders, Phys. Rev. D 94 (2016) 015001 [arXiv: 1605.00658] [INSPIRE].

[27] R. Mahbubani and J. Zurita, To appear.

[28] ATLAS collaboration, Search for charginos nearly mass degenerate with the lightest neutralino based on a disappearing-track signature in pp collisions at $\sqrt{s}=8 \mathrm{TeV}$ with the ATLAS detector, Phys. Rev. D 88 (2013) 112006 [arXiv:1310.3675] [INSPIRE]. 
[29] CMS collaboration, Search for disappearing tracks in proton-proton collisions at $\sqrt{s}=8 \mathrm{TeV}$, JHEP 01 (2015) 096 [arXiv:1411.6006] [INSPIRE].

[30] S.D. Thomas and J.D. Wells, Phenomenology of Massive Vectorlike Doublet Leptons, Phys. Rev. Lett. 81 (1998) 34 [hep-ph/9804359] [INSPIRE].

[31] M. Cirelli, N. Fornengo and A. Strumia, Minimal dark matter, Nucl. Phys. B 753 (2006) 178 [hep-ph/0512090] [INSPIRE].

[32] J. Alwall et al., The automated computation of tree-level and next-to-leading order differential cross sections and their matching to parton shower simulations, JHEP 07 (2014) 079 [arXiv: 1405.0301] [INSPIRE].

[33] M.L. Mangano, M. Moretti, F. Piccinini, R. Pittau and A.D. Polosa, ALPGEN, a generator for hard multiparton processes in hadronic collisions, JHEP 07 (2003) 001 [hep-ph/0206293] [INSPIRE].

[34] T. Sjöstrand, S. Mrenna and P.Z. Skands, PYTHIA 6.4 Physics and Manual, JHEP 05 (2006) 026 [hep-ph/0603175] [INSPIRE].

[35] M. Cacciari, G.P. Salam and G. Soyez, FastJet User Manual, Eur. Phys. J. C 72 (2012) 1896 [arXiv:1111.6097] [INSPIRE].

[36] E. Conte, B. Fuks and G. Serret, MadAnalysis 5, A User-Friendly Framework for Collider Phenomenology, Comput. Phys. Commun. 184 (2013) 222 [arXiv:1206.1599] [INSPIRE].

[37] E. Conte, B. Dumont, B. Fuks and C. Wymant, Designing and recasting LHC analyses with MadAnalysis 5, Eur. Phys. J. C 74 (2014) 3103 [arXiv:1405.3982] [INSPIRE].

[38] B. Dumont et al., Toward a public analysis database for LHC new physics searches using MADANALYSIS 5, Eur. Phys. J. C 75 (2015) 56 [arXiv:1407.3278] [inSPIRE].

[39] W. Beenakker, M. Klasen, M. Krämer, T. Plehn, M. Spira and P.M. Zerwas, The production of charginos/neutralinos and sleptons at hadron colliders, Phys. Rev. Lett. 83 (1999) 3780 [Erratum ibid. 100 (2008) 029901] [hep-ph/9906298] [INSPIRE].

[40] ATLAS collaboration, Search for winos using a disappearing track signature in ATLAS, ATL-PHYS-SLIDE-2017-104 (2017).

[41] P. Schwaller, D. Stolarski and A. Weiler, Emerging Jets, JHEP 05 (2015) 059 [arXiv: 1502.05409] [INSPIRE].

[42] C.H. Chen, M. Drees and J.F. Gunion, A nonstandard string/SUSY scenario and its phenomenological implications, Phys. Rev. D 55 (1997) 330 [Erratum ibid. D 60 (1999) 039901] [hep-ph/9607421] [INSPIRE].

[43] C.H. Chen, M. Drees and J.F. Gunion, Addendum/erratum for 'searching for invisible and almost invisible particles at $e+e$ - colliders' [hep-ph/9512230] and 'a nonstandard string/SUSY scenario and its phenomenological implications' [hep-ph/9607421], hep-ph/9902309. 